\title{
Toward a framework for the analysis and design of educational games
}

\author{
Vincent Aleven*, Eben Myers $\dagger$, Matthew Easterday*, Amy Ogan* \\ * Human-Computer Interaction Institute \\ Carnegie Mellon University \\ Pittsburgh, PA \\ + Etcetera Edutainment, Inc. \\ Pittsburgh, PA \\ USA \\ USA \\ aleven@cs.cmu.edu,emyers@etceteraedutainment.com, \\ mwe@andrew.cmu.edu,aeo@adrew.cmu.edu
}

\begin{abstract}
We describe and illustrate the beginnings of a general framework for the design and analysis of educational games. Our students have used it to analyze existing educational games and to create prototype educational games. The framework is built on existing components: a method for precisely specifying educational objectives, a framework for relating a game's mechanics, dynamics, and aesthetics, and principles for instructional design grounded in empirical research in the learning sciences. The power of the framework comes from the components themselves, as well as from considering these components in concert and making connections between them. The framework coordinates the many levels at which an educational game must succeed in order to be effective. We illustrate the framework by using it to analyze Zombie Division and to generate some redesign ideas for this game.
\end{abstract}

\section{Introduction}

Creating a game (digital, non-digital, or otherwise) that is both fun and educational is a significant challenge. However, very little has been written about how to design effective educational games, a stark contrast to the large number of books about game design published in the last decade [1, 2, 3, 4].

In this paper we present a framework that helps designers to identify and analyze the important design choices that need to be made while creating an educational game. By analyzing an educational game from multiple analytical angles, the framework helps the designer to coordinate the many levels at which an educational game must succeed in order to meet its objectives. Over the last two years, graduate and undergraduate students in Carnegie Mellon University's Design of Educational Games course have used the framework both to analyze and design educational games.

The framework consists of 3 components: learning objectives, MDA, and Instructional Design Principles, as well as a strategy for combining them. Familiar to instructional designers, Bloom's Revised Taxonomy helps in characterizing the learning objectives that the game targets, which can be surprisingly helpful in making sure that the game meets its goals. For many game designers, the MDA framework [5] describes and connects three interrelated levels: mechanics (M), dynamics (D), and aesthetics (A). Research-based Instructional Design Principles promote thinking about whether the game supports learning in ways that are consistent with empirical research on instruction and robust learning. In addition to these "standard" components, we present a simple strategy for how to effectively use the components in concert. This framework may be thought of as a web; tugging on one part of the web (i.e., one aspect of a game's design) has the potential to disturb other aspects to varying degrees, sometimes in a good way, sometimes in a way that harms the intended outcome. The framework helps the designer to keep the whole picture in mind. While the framework components themselves are not novel, the use of them in concert to create educational games $i$, to the best of our knowledge, as are these heuristics for thinking "across the components."

Our main claim in this paper is that the analytical framework is useful both for analysis of educational games and for their design. We expect the first part of this claim to be relatively uncontroversial, and expect the second part perhaps to spur more discussion. In this paper we illustrate the framework, and present a case study in applying it to the analysis of an existing educational game, Zombie Division [6]. We also illustrate its use to generate and evaluate redesign ideas 
for the same game, thereby illustrating the two-part claim made above.

\section{An illustration using Zombie Division}

To illustrate the framework, we apply it to Zombie Division, an educational game created by Jacob Habgood and Shaaron Ainsworth at the University of Nottingham to complement classroom instruction. According to its creators, "The game integrates mathematics into the core-mechanic of an actionadventure game through a combat system in which opponents are mathematically divided in order to defeat them" [6].

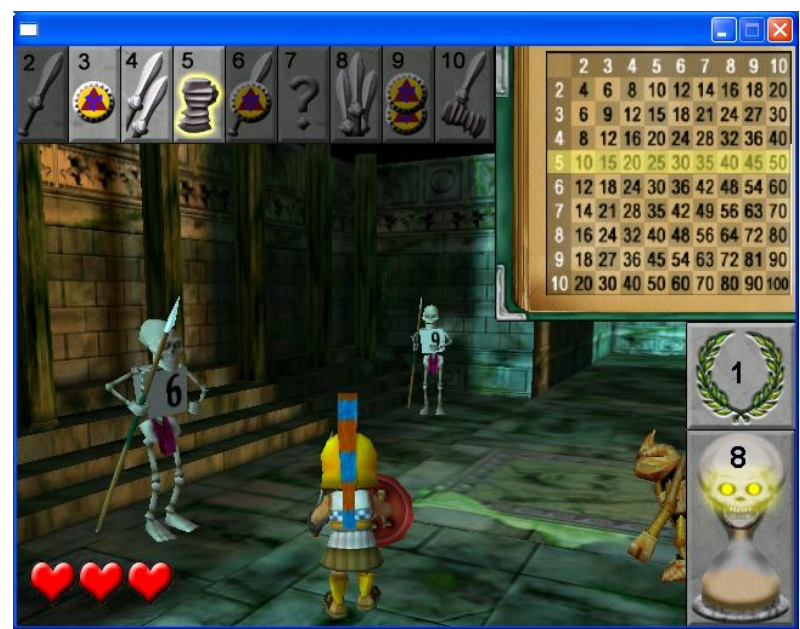

Figure 1: Zombie Division

Zombie Division is designed to help 7- and 8-year old student/players develop both a conceptual understanding of division as the inverse of multiplication and skill and fluency in making divisibility judgments with whole numbers. The game is a $3 \mathrm{D}, 3^{\text {rd }}$-person action game. The student/player takes on the identity of Matrices, an ancient Greek warrior of small stature whose mission it is to rid a dungeon of zombies ${ }^{1}$. Matrices wields a set of up to three weapons such as a sword, a shield, an iron fist, etc, numbered from 2 to 10 that varies by level (see the top of the screen in Fig. 1). A Zombie will only die when it is attacked with a weapon whose number is a divisor of the number on the Zombie's chest. Thus, a successful attack requires a judgment as to whether the zombie's number can be divided evenly by any of the available weapons. Attacks with any weapon that is not a divisor of the zombie's number will be unsuccessful and cause the loss of health; when all health is lost, the student/player starts the level from the beginning.

\footnotetext{
${ }^{1}$ Specifically, the student must defeat 20 zombies at each of the game's 20 or so levels.
}

Zombie Division also includes a bone-dragging exercise in the system's help screen, which aims at developing an understanding of division as dividing a number into equal parts. Here the players must sort a large pile of bones, one by one, into equal smaller piles, as a way of building up a conceptual understanding of the division operator.

\section{The framework}

We now describe each of the three components of the framework individually, and then discuss how the framework supports a game analyst or designer in applying them in concert with one another.

\section{Component 1. Learning objectives}

The first component of the framework is a specification of learning objectives. A clear specification of learning objectives helps designers ensure that the game they create actually meets an intended and coherent set of educational goals, rather than an opportunistic and less coherent set of educational goals. For example, it helps them avoid creating games that address only simple instances of a targeted cognitive skill, or games that assume prior knowledge that the student/player is not likely to have; or games that include a mechanic that enables the student/player to avoid hard learning problems embedded within the game. Further, it is hard to see how a designer of educational games could create a game that is truly educationally effective if he/she is not clear what the educational goals are [7].

Specifying the learning objectives requires answering three questions:

1. (Prior knowledge) What knowledge or skills do student/players need to have before starting the game?

2. (Learning and retention) What knowledge or skills can student/players reasonably be expected to learn from the game?

3. (Potential transfer) What knowledge and skills might they learn that go beyond what they actually encountered in the game?

Answering these questions requires: (a) providing a written specification of the knowledge and skills, (b) providing examples of tasks by which a student/player will improve the given knowledge and skills and (c) categorizing the knowledge, skills and tasks in the previous steps using Bloom's Revised Taxonomy [7]. The use of this taxonomy helps in achieving a detailed understanding of the learning objectives and for considering whether the objectives should be extended to include higher-level cognitive processes. 
Following our framework, the learning objectives of Zombie Division can be analyzed as follows. First, in order to make any headway at all student/players must have the following prior knowledge (question 1):

- Some understanding of multiplication, since the game reinforces a notion of division as the inverse of multiplication through the use of a 10x10 multiplication table. For example, the student/player must have some notion of what it means that $7 \times 8=56$.

- The student/player must have a basic notion of division as a way of understanding how (some) larger numbers can be built through multiplication of smaller numbers.

Question two asks the designer to consider learning and retention. By playing Zombie Division, the student/player is likely to acquire a better understanding of, and fluency with, making divisibility judgments with dividends less than 100 and divisors less than 10. Examples of problems at which the Zombie Division student/player will likely improve are:

- Dividend-based example by which of these divisors can you divide 42: $3,5,6$, or none of the above? The student/player is confronted with this problem type when they encounter Zombie 42 while carrying weapons 3,5 , and 6 .

- Dividend-based prime example: by which of these divisors can you divide $41: 3,5,6$, or none of the above? In this case, the answer is none of the above, as 41 is a prime. In the game, the student/player should leave this zombie alone.

- Divisor-based example: which of these numbers can be divided by $6: 15,26,48$, or none of the above? This reflects the situation where a student/player carrying weapon 6 walks into a room with three zombies with the respective dividends.

These examples fit under the categories "Applying Procedural Knowledge" in Bloom's Revised Taxonomy.

It is likely also that student/players will become better at doing division problems (e.g., what is 42 divided by 3 ?), even though the game does not pose problems in exactly this format (i.e., the student/player never has to actually enter the quotient of two numbers in order to succeed in the game). There are (at least) two ways in which student/players nonetheless may get better at this type of problem: First, he/she may make divisibility judgments by actually doing the division (e.g., 42 is divisible by 3 because $42 \div 3=14$ ). Second, the game provides many examples of solved division problems: When an attack on a skeleton succeeds, the game will carry out the division for the student/player; the skeleton visually divides into a number of pieces equal to the divisor ${ }^{2}$, which, each marked with the quotient, then disperse by rising through the ceiling.

Finally, with respect to question 3 , transfer, one might speculate that the game helps student/players learn prime numbers. These concepts are related to the division and divisibility concepts and skills that the game supports. Strictly speaking, the game does not require knowledge of prime factors and prime numbers, nor does it reward student/players for mastering them. Nonetheless, a bright student/player may learn these skills and concepts by playing the game. For example, she may notice that some zombies cannot be divided at all, and reflect upon this fact. However, the game provides no direct support for learning these skills. We therefore categorize these kinds of problems as transfer [8], meaning that they go beyond what is practiced in the game. Since predicting transfer is difficult in general, it is hard to say whether and how much student/players will learn about prime numbers.

Armed with this kind of detailed understanding of what a game is designed to accomplish, the designer of an educational game is in a better position to create an effective game, or to generate good redesign ideas.

\section{Component 2: MDA}

The second component of our framework is, itself, a framework. The MDA framework [5] helps game analysts and designers think about games in terms of three mutually-dependent layers. The (M)echanics of a game are the basic components out of which the game is built: the materials, rules, explicit goals, basic moves, and control options available to the players. The (D)ynamics of the game are the behaviors that result when applying the game's mechanics with player input during game play. The MDA framework does not provide a taxonomy of game dynamics. It is up to the designer or analyst to invent the terms and concepts needed to characterize the dynamics of a given game. The (A)esthetics of a game, finally, capture the subjective experience of the player, the emotional response or pleasure that the game is designed to evoke. The MDA framework does provide a taxonomy of aesthetic elements commonly targeted in games, which comprises 8 items: Sensation, fantasy, narrative, challenge, fellowship, discovery, expression, and submission. This non-exhaustive taxonomy provides a concrete and nuanced way of talking about the

\footnotetext{
${ }^{2}$ This is the somewhat tongue-in-cheek way in which the mathematics and the game's core mechanic are thematically connected: the mathematical breaking down of numbers achieves the physical breaking down of enemies.
} 
pleasures resulting from games, in an attempt to move beyond vague terms such as "fun" and "game play." The three levels are related in the sense that a game's mechanics give rise to its dynamics, which in turn evoke a certain aesthetic. However, as a game is being designed and developed, the three levels need not (and should not) be considered just in this order. While a player's experience of a game is typically at the aesthetic level, a designer only has control over the mechanics. The MDA framework allows a designer to articulate aesthetic goals and make reasoned choices at the mechanical level to support that aesthetic outcome. Dynamics are an important middle ground where the designer's mechanical choices meet with players input. For example, the designer may have a certain aesthetic in mind (e.g., challenge), consider possible dynamics that may give rise to this aesthetic (e.g., time pressure), and try out different mechanics that may give rise to this dynamic (e.g., use of a timer, or of parallel play by multiple players with bonus points for whoever finishes their move first).

Of the eight aesthetic elements in the MDA taxonomy, challenge is most clearly associated with Zombie Division. The central focus of the game, and a central influence shaping a student/player's aesthetic experience, is the challenge of taking on and defeating a large number of zombies. Interestingly, the learning content adds to the feeling of challenge. The mathematics needed to defeat zombies are not easy for the student/players in the target population. A number of special game mechanics further add to the challenge. For example, at some levels zombies emerge from a hole in the floor in quick succession, and come running at Matrices. This mechanic forces the player to react quickly, which temporarily increases the challenge of the game. A secondary aesthetic element associated with the game is fantasy. Not only does the game put the student/player in a position where they assume the identity of an imaginary Greek warrior, it also is situated in that staple of fantasy worlds, the dungeon where the main action is battling monsters.

As mentioned, a game's dynamics are the behaviors that result within the game world from actions sanctioned by the game's mechanics. The predominant dynamic supported by Zombie Division is a somewhat deliberate style of suspenseful hunting. We prefer to characterize the engagements with the zombies as hunting, rather than combat, since the zombies are not very combative. They succumb after a single strike with an appropriate weapon, and rarely attack out of their own volition. A key aspect of the game's deliberate hunting dynamic is that student/players often experience mild time pressure, over which they have some control. On one hand, it is hard not to feel pressured when close to a zombie, and especially when surrounded by zombies, as happens occasionally. On the other hand, a student/player can oftentimes avoid the pressure by walking out of the room, or by stopping when seeing a zombie in the distance. Great time pressure occurs occasionally, namely, at the levels where zombies emerge from the floor, as described above. At other times, the pace is very slow, namely, when players engage in the bone-dragging exercise.

A key aspect of our framework is to analyze how the game's mechanics, dynamics, and aesthetics relate to its learning objectives (or learning content). We typically ask our students to discuss these connections when analyzing or designing a game. The connection between Zombie Division's mechanics and its learning content has been discussed extensively in the writings by Habgood and Ainsworth [6]: the learning content is tightly integrated into the game's core mechanic. In order to attack a zombie with an appropriate weapon, a student/player must make a divisibility judgment. A scientific evaluation study confirmed that this tight integration between learning and core mechanic leads to better learning and greater motivation on the part of the student/player, compared to simply alternating between math and game play without any connection between them [6].

The relation between the game's dynamics and the learning content must be carefully considered, as certain dynamics may be more suitable for certain types of learning content. A fast-paced game dynamic may be more suitable for learning the fluent execution of a skill, whereas a slower and more deliberate dynamic may be more suitable if the game's learning objectives require that the student/player makes sense of the learning content embedded in the game. As discussed, most of the time Zombie Division generates a somewhat deliberate, slower-paced dynamic, but occasionally switches into a faster-paced more hectic mode, and occasionally switches into a very slowpaced, possibly dull mode. Our guess is that this differentiation was motivated largely by the different educational goals that the game aims to support. The medium pace of the game allows student/players to think while executing the divisibility problems, while perhaps using the multiplication table that is always on the screen. The faster-paced activities are no doubt meant to build fluency with divisibility judgment and division skill. Finally, we think that the slow pace (and resulting tedium) of the bone-dragging exercises has an educational purpose as well. The tedium may be meant to help bring the point across that division is a powerful operator that does away with the need for repetitive 
sorting of "stuff" into piles. In other words, the tedium might be a way of helping student/players see the need for, and power of, the new mathematical idea they are learning. The danger may be that the tedium causes student/players not to pay attention, and we are not fully convinced that the game strikes the right balance. However, it is an interesting example of employing a particular aesthetic (tedium) to reinforce an educational objective.

Finally, how do the game's aesthetics relate to learning? Perhaps the fantasy aesthetic is mainly there for motivational purposes, allowing the Greek Warrior fantasy to be effective without relating directly to division. The challenge aesthetic is associated with LLL\#14 "Desirable Difficulties:" making initial knowledge acquisition more difficult, yet improving retention and transfer. Conversely, the learning content itself adds to the challenge of killing zombies.

\section{Component 3: Instructional principles}

The third main component of our framework is the use of research-based principles for instructional design. Many such collections exist. These collections are an increasingly popular way in which learning sciences researchers summarize and communicate the results of their research to instructional designers and instructors (for a list of lists, see http://www.learnlab.org/research/wiki/index.php/Instru ctionalPrinciples). We draw on a number of collections of principles that have been widely published, as a way of bringing the learning sciences into the design of educational games. A key assumption is that instructional design principles that have been established in other types of learning environments will carry over to the design of educational games, and can help create games that are effective, educationally.

First, the Multi-Media Principles [9] by Mayer and Moreno provide research-based guidelines for creating instructional materials that combine verbal and visual information. Second, the Cognitive Tutor principles [10] derive from research on intelligent tutoring systems. They focus on learning environments that provide step-by-step support as a learner practices a complex cognitive skill. Clearly, some educational games target such skills, including Zombie Division. Third, the Life-Long Learning principles are a compilation of 25 principles culled from learning science research (http://www.psyc.memphis.edu/ learning/principles/). Finally, Jim Gee's 36 principles of game-based learning describe how learning is supported within standard, commercial, off-the-shelf video games [11]. Unlike the other sets of learning principles, Gee's principles are not supported by quantitative experimental studies, but they are the only set that we know of that is specific to learning in video games. We anticipate that they will be helpful in designing games for explicit educational goals. Although there is some overlap between the four collections of principles, they all have a somewhat different focus, which is why we include all four collections in our framework.

As we analyze games, we find it helpful to consider which instructional principles they implement. The principles provide a way to think and talk about how a game supports learning, and whether it does so in ways consistent with learning sciences findings and recommendations. We have also found it helpful to identify principles that a game might violate or ignore. As we discuss further below, this kind of analysis can lead to redesign ideas focused on the educational effectiveness of a game. We add that there is no expectation that any game, or any instructional intervention, covers all the principles. Rather, one looks for a coherent story as to how the game supports learning, backed up by principles, and one considers whether and how the game might be extended to support learning even more effectively through the use of additional principles.

Zombie Division implements a number of principles from the sources listed above. Perhaps most strikingly, the game provides ample opportunity for practice of the targeted math skills. There are many zombies to be slain, each requiring application of math skills. Zombie Division quite comprehensively covers the targeted space of divisibility/division problems, those with dividends less than 100 and divisors less than 10. Thus, the game implements Gee $\# 12^{3}$ : "Learners get lots and lots of practice in a context where the practice is not boring." Further, the game provides feedback and guidance. It provides immediate feedback on the success on each opportunity to apply the targeted math skills. The zombie either strikes back (negative feedback) or it succumbs (positive feedback). When it succumbs, it divides into pieces that slowly float upwards and disappear, each of which is marked with the quotient. Thus, the game implements CT \#6: "Provide immediate feedback on errors" as well as LLL \#13: "Learning wrong information can be reduced when feedback is immediate." The animation of the zombie's pieces marked with the quotient is a form of positive feedback, but it can equally be seen as a source of worked examples of the targeted division skills.

\footnotetext{
${ }^{3}$ To indicate the source of the principle, we use the following abbrevations: $\mathrm{MM}=$ Multi-Media Principles; $\mathrm{CT}=$ Cognitive Tutor Principles; LLL = Life-Long Learning Principles; Gee = Jim Gee's Principles of Game-Based Learning.
} 
Thus, the game reflects LLL \#11: “An understanding of an abstract concept improves with multiple and varied examples."

In addition to supporting student/players in gaining fluency with the targeted math skills, Zombie Division provides a help screen with two forms of help meant to communicate a conceptual view of division. The help screen offers the bone-sorting exercises described above and help with the use of the multiplication table to make divisibility judgments. Thus, Zombie Division instantiates CT \#3 "Provide instruction in the problemsolving context." This principle recommends that problem-solving practice be accompanied by "declarative" instruction about relevant concepts (e.g., explanations, or interactive activities with a conceptual focus). The provision of help by the system also reflects Gee \# 27 "Explicit Information On-Demand and Just-in-Time Principle: The learner is given explicit information both on-demand and just-in-time, when the learner needs it or just at the point where the information can best be understood and used in practice." The bone-sorting exercises in Zombie Division are always available on demand, and are also offered proactively by the game after the student/player makes an error. However, the student/player can easily opt out, which may be less than ideal, given evidence that students in interactive learning environments often do not use on-demand help facilities effectively [12].

Finally, the fact that the math in the game gradually gets to be more challenging as the player advances through the levels is an instantiation of LLL \#21 "Goldilocks Principle: Assignments should not be too hard or too easy, but at the right level of difficulty for the student's level of skill or prior knowledge." A similar idea is expressed in Gee \#14 "Regime of Competence Principle: The learner gets ample opportunity to operate within, but at the outer edge of, his or her resources, so that at those points things are felt as challenging but not undoable."

As mentioned, when analyzing a game or other type of learning environment, one looks for a coherent story, supported by principles, as to how the game supports learning. One does not look for complete coverage of all principles. Measured by this yardstick, Zombie Division succeeds admirably, an impression that is confirmed by the evaluation studies done by Habgood and Ainsworth [6].

\section{Combining components to (re)design}

So far, we have illustrated each of the main components of our framework. In this section, we illustrate, again using Zombie Division as our running example, a strategy for thinking across the components of the framework, and illustrate how it can often lead to useful design or redesign ideas. The point is not to present spectacular improvements to Zombie Division. We acknowledge that the game's designers may have considered many of the same ideas while creating Zombie Division. Rather, the point is to illustrate how the framework supports useful thinking about design. Essentially, one can use each of the three main components of the framework as a focus for brainstorming during initial design. One can then consider whether ideas generated while focusing on one component work well within the other components of the framework. This simple strategy can help make sure that ideas work well within all three components, and more broadly, that a game succeeds with respect to all components.

Learning objectives focus. One approach to generating design ideas is to focus on the game's learning objectives, generating ideas for how they might be enhanced. One should then consider how each of the MDA levels can accommodate these additional learning objectives, and one should also consider the subsequent effect on any instructional principles supported by the game. A useful way to expand the learning objectives for a given game is to look at the knowledge and skills that were originally considered as "transfer," and to consider whether they can be brought into the game.

For example, learning about prime numbers is not strongly supported in Zombie Division, which is why, as discussed above, we consider it to be a form of transfer. We might consider how learning about prime numbers could be added to the game's learning objectives and integrated into the game. We can turn to the MDA component of the framework in concert with an understanding of the learning objectives to investigate what game mechanic might best help student/players in learning about prime numbers. If the critical goal is an understanding that prime numbers are "different" from other numbers, one could include a special weapon for prime numbers. One of our students suggested a hammer that "pulverizes" prime-numbered zombies (though not other zombies) to simulate division with quotient 1 , an idea that is nicely compatible with the game's fantasy aesthetic. An alternative way of bringing the objective of learning prime numbers in line with the game's fantasy, primenumbered zombies could remain "immune" to division by Matrices' weapons (they currently are immune in this manner), but could be jailed in a remote cell in the dungeon, to emphasize that prime numbers cannot be divided. The added task of identifying and eliminating 
prime numbers would support and extend the aesthetic experience of challenge.

The designer would still need to consider how the contemplated change relates to relevant instructional principles. For example, the designer might focus on principles that are currently instantiated in the game, such as those discussed above. She might make sure that the prime-numbered zombies would show up frequently enough to provide sufficient opportunities for practice (Gee \#12: Practice Principle). She might also make sure, one way or another, that the game selects appropriately challenging prime numbers (LLL \#21: Goldilocks Principle). Thus, the example illustrates thinking across the components of the framework. A contemplated change in learning objectives is achieved with new mechanics that are nicely compatible with the game's fantasy aesthetic, and that can be realized in ways compatible with important learning principles.

MDA focus. To design or redesign a game, one might think in terms of MDA in order to generate ideas that enhance or change the game's aesthetics and perhaps dynamics. One must then verify that they support or strengthen the game's learning objectives, and that they align well with instructional principles. For example, the dungeon in Zombie Division suggests the idea of a discovery aesthetic; as mentioned, discovery is one of the eight aesthetic pleasures in the MDA framework. Currently, the discovery aesthetic of Zombie Division is not very strong. The dungeon is not very extensive, and the only thing to be found (except zombies) is the occasional key needed to open doors. The discovery aesthetic could be enhanced if there was a more interesting maze, with some dark secret to be discovered that the zombies are trying to protect. This MDA improvement could be designed to support the educational objectives if the secret discovery was helpful mathematically: perhaps the zombies are holding hostage a group of ancient Greek mathematicians who know about shortcut strategies for making divisibility judgments (e.g., even numbers are divisible by 2 , numbers ending in 0 or 5 are divisible by 5 , etc.). Upon being freed by Matrices, they are very forthcoming with guidance about these strategies, out of gratitude, but also because these strategies will enable Matrices to strike back at their captors. This change would add additional learning objectives to the game in the form of shortcut strategies, which should be evaluated for appropriateness in the instructional context. In our example, it seems entirely appropriate and desirable to add the shortcut strategies as new learning objectives. The contemplated change should also be evaluated with respect to relevant learning principles. The notion of Greek mathematicians who are eager to share mathematical "secrets" immediately brings to mind Gee \#27 "Explicit Information OnDemand and Just-in-Time Principle." Interestingly, this principle does force the game designer to consider the timing of the help. Our game designer might want to make sure that the shortcut strategies should be introduced neither too early nor too late, that is, after the student/player has developed a good conceptual understanding of division but not so late that there is little opportunity to use and practice them within the game. Further, in line with the notion of providing help just in time, one would like the Greek mathematicians to communicate a given shortcut strategy to the student/player at the very moment that she can apply it in the game. But what mechanics will make this possible? Will the mathematicians accompany Matrices as he roams the dungeon, and then, when a certain zombie is spotted, communicate the shortcut strategy applicable to this zombie? Or, conversely, will the mathematicians lead Matrices to particular zombies that can be dispatched with a certain shortcut strategy? No doubt, satisfactory solutions can be found to these questions. The key point is that the example again illustrates the value of thinking across the components of the framework. A change aimed at enhancing the game's discovery aesthetic naturally leads to an additional learning objective, which when considered in light of important learning principles, leads the designer to consider further game mechanics.

Instructional principles focus. A final source of redesign ideas may be to consider whether additional instructional principles can be brought into play. One strategy to employ when considering the principles component of the framework is to look for "near misses," principles that seem relevant to the game and to the principles it does implement. Ideas generated in this manner also need to be considered from the viewpoint of the other components within the framework, to ensure that the aesthetic experience of play is not changed unintentionally or is changed in a beneficial way. Further, it is important to keep track of the effect (if any) that the change will have on the game's learning objectives and avoid undesirable changes.

A principle that seems relevant to Zombie Division is LLL \#5 "Spacing Effect. Spaced schedules of studying and testing produce better long-term retention than a single study session or test." Although game designers have little control over when kids play a game they design, they do have some control over the spacing of practice items within the game; that is, they control when certain practice items re-occur. Zombie 
Division may be even more effective if it spaced out practice with each divisor or dividend across the game according to current spacing theory. To implement this idea, a new mechanic is needed by which the available set of weapons changes more often than it currently does. (It is currently fixed at each level.) The game may also need to make adaptive decisions as to which Zombies will inhabit a room. It is not clear that the dynamics of the game would change greatly, but possibly, the challenge aesthetic would be enhanced, as players encounter division tasks that are more finely tuned to push their skill level. The learning objectives would remain the same.

Further, it would be interesting to think about how the Gee \#14 Regime of Competence and LLL \#21 principles, discussed above, could be supported even more strongly, for example by using adaptive techniques from intelligent tutoring systems [13]. That is, the game might adjust the rate at which the challenge level increases on an individual basis, based on its assessment of how fast each individual student/player is learning. Here again, the challenge aesthetic might be enhanced. The game may also adaptively introduce the learning objectives previously identified as transfer to increase the level of challenge.

The specific redesign ideas we have presented here are not as important as the process by which they were generated, namely, to think across the components of the framework.

\section{Conclusion}

We present a framework for the design of educational games, based on three main components, known to game designers and instructional designers. Each component provides its own analytical angle. Basic assumptions underlying the framework are that an educational game development project is more likely to succeed if the learning objectives of the game have been clearly established early on in the development process, if the designers have carefully thought about how the game's desired aesthetic can be grounded in the game mechanics, through the game's dynamics, and if the game observes well-established instructional design principles. A designer of educational games needs to consider almost constantly how he or she can make the components work in concert. An educational game has to succeed on two fronts: as an educational tool and as a fun game. Fun is judged through success in achieving the aesthetic goals. The mechanics and dynamics are merely tools for getting there. The learning principles work predominantly at the level of the dynamics and mechanics. The trick is keeping aesthetics in line while tweaking the mechanics and dynamics to work in accordance with instructional principles to meet the game's learning goals.

As our running example illustrates, the framework is not just a useful analytical tool, but can also help enhance the creativity of the designer of educational games, in a modest way, by suggesting some useful directions when brainstorming game ideas, and by suggesting that these ideas be considered from both educational and game design angles. In our course, students have used and are using the framework as they analyze games, and as they design educational games for their projects.

To summarize, the framework helps a designer be deliberate and intentional about the design choices they make. We hope to have illustrated that the framework helps in thinking about whether additions or modifications to the game's mechanics that are intended to make the game more fun also work at an educational level; or at least to spot situations where design decisions may need to trade off educational objectives and fun objectives, which sometimes is a reasonable thing to do.

\section{Acknowledgements}

Jennifer Olson, a student in our course "Design of Educational Games" contributed the prime number hammer design idea described in this paper.

\section{References}

[1] Fullerton, T., Swain, C., \& Hoffman, S. (2004). Game design workshop. San Francisco, CA: CMP books.

[2] Habgood, J., \& Overmars, M. (2006). The game maker's apprentice: Game development for beginners. Apress.

[3] Salen, K., \& Zimmerman, E. (2003). Rules of play: Game design fundamentals. Cambridge, MA: MIT Press.

[4] Schell, J. (2008). The art of game design: A book of lenses. Morgan Kaufmann.

[5] Hunicke, R., LeBlanc, M., \& Zubek, R. (2004). MDA: A formal approach to game design and game research. In D. Fu, S. Henke, \& J. Orkin (Eds.), Challenges in game artificial intelligence, papers from the 2004 AAAI workshop (pp. 1-5). Technical Report WS-04-04. Menlo Park, CA: The AAAI Press.

[6] Habgood, M. P. J., \& Ainsworth, S. E. (under review). Does intrinsic integration matter? Exploring the value of intrinsic integration in educational games. Manuscript submitted for publication.

[7] Anderson, L. W., Krathwohl, D. R., Airasian, P. W., Cruickshank, K. A., Mayer, R. E., Pintrich, P. R., et al. (2001). A taxonomy for learning, teaching, and assessing: A revision of Bloom's taxonomy of educational objectives (Abridged ed.). New York: Longman. 
[8] Singley, M. K., \& Anderson, J. R. (1989). The transfer of cognitive skill. Cambridge, MA: Harvard University Press.

[9] Mayer, R. E., \& Moreno, R. (2003). Nine ways to reduce cognitive load in multimedia learning. Educational Psychologist, 38(1), 43-52.

[10] Anderson, J. R., Corbett, A. T., Koedinger, K. R., \& Pelletier, R. (1995). Cognitive tutors: Lessons learned. The Journal of the Learning Sciences, 4(2), 167-207.

[11] Gee, J. P. (2007). Good video games and good learning: Collected essays on video games, learning and literacy. New York: Peter Lang.

[12] Aleven, V., Stahl, E., Schworm, S., Fischer, F., \& Wallace, R. (2003). Help seeking and help design in interactive learning environments. Review of Educational Research, 73(3), 277-320.

[13] Woolf, B. P. (2008). Building intelligent interactive tutors: Student-Centered strategies for revolutionizing $e$ learning. Morgan Kaufmann. 